by different coloured light. By generating overlapping images in different colours and compiling them, the system can produce threedimensional images that have a spatial resolution ten times better than confocal images in each dimension, Zhuang says. Nikon's instrument, called N-STORM, should be available by May 2010.

\section{Technology tweaks}

The pioneers of super-resolution microscopy are continuing to improve their methods with better sample preparation, a few strategically placed pieces of hardware and more sophisticated algorithms.

One way to achieve greater resolution in fixed samples is to cut thinner slices of tissue. Some groups, including Zhuang's, are collaborating to combine STORM with array tomography, which involves embedding fixed tissue in resin and cutting ultrathin (50-200 nm) slices. "Array tomography is a potential adjunct to all the super-resolution methods," says Stephen Smith of Stanford University in Palo Alto, California, who first described the method. Using this combination, his group has achieved a 14 -fold improvement in axial resolution, and twofold improvements in the each of the two dimensions, compared with confocal imaging.

Other combination strategies are possible. For example, two-photon excitation microscopy, an established method that allows researchers to see further into tissues, can be combined with STED microscopy. Earlier this year, Hell's group used the technique on

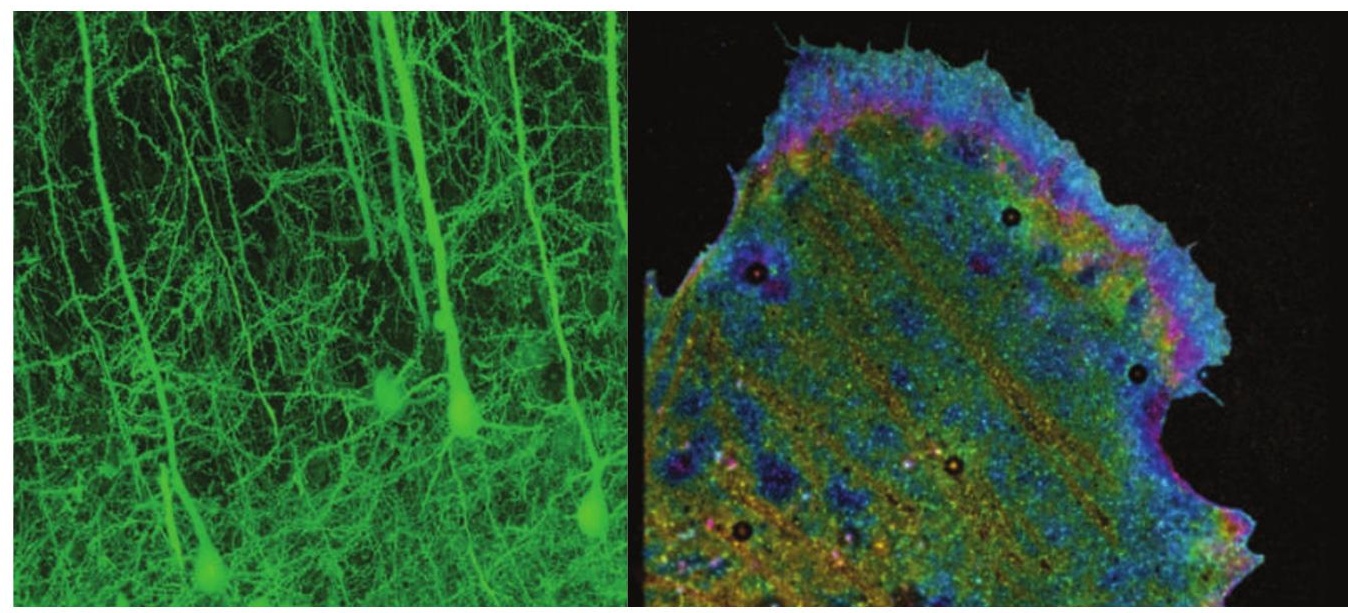

New microscopy techniques are showing structures (pyramidal neurons, left and a U2OS bone cell, right) in unprecedented detail.

mammalian cell nuclei tagged with fluorescent nanoparticles, obtaining a resolution of less than $70 \mathrm{~nm}$ in the focal plane ${ }^{1}$.

Bernardo Sabatini, a neurobiologist at Harvard Medical School in Boston, Massachusetts, used the same combination to image slender neuronal projections and dendritic spines in brain slices, which are not visible using conventional methods ${ }^{2}$. Sabatini and his team built the system themselves. They created the ring shape in the depletion laser using a phase plate to split the laser light so that one part served as the central spot and the other part as the ring. The result was a three-fold improvement in spatial resolution over normal two-photon techniques.
Advances in resolution have not been

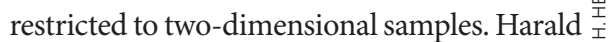
Hess and his colleagues at Howard Hughes Medical Institute's Janelia Farm Research Campus in Virginia have modified PALM to do 3D imaging by adding an interferometer and a second objective lens ${ }^{3}$. Called iPALM, this method can pinpoint fluorescent labels to within 10-20 nm in three dimensions. However, the samples must be around 225-250 $\mathrm{nm}$ thick, and ensuring that the extra optics needed are perfecty aligned is a time-consuming task. As a result, iPALM might prove challenging to commercialize, Hess says.

Groups led by Moerner and Rafael Piestun at the University of Colorado, Boulder, have

\title{
BREAKING THE LIGHT BARRIER
}

In 1873, German physicist Ernst Abbe proposed that diffraction fundamentally limited the resolution that any microscope could achieve to around half the wavelength of light. And, despite many advances, microscopes didn't threaten to challenge this law of physics for more than a century. Stefan Hell, now a director of the Max Planck Institute for Biophysical Chemistry in Göttingen, Germany, was the first to show that the diffraction limit could be beaten.

Hell, while a postdoc at the University of Turku in Finland in the 1990s, thought that, with the right lasers, he could activate a fluorescent spot and then shrink it by superimposing a larger, hollow beam of light to deplete all the light emission except for that at the centre of the spot. He called the technique stimulated emission depletion (STED) microscopy. Although many physicists were initially sceptical of Hell's ideas, by 2000 he had used STED to produce the first nanoscale fluorescence images ${ }^{10}$.

Super-resolution microscopy has blossomed since, allowing researchers to see cellular processes unfolding at nanometre scales. "This is something that the field has desired since people first started looking through light microscopes," says Jan Liphardt, a biophysicist at the Lawrence Berkeley National Laboratory in California.

Since Hell's work, the field has been boosted by other groups, including those of Eric Betzig, a physicist at the Howard Hughes Medical Institute's Janelia Farm Research Campus in Virginia and Jennifer Lippincott-Schwartz, a cell biologist at the National Institutes of Health in Bethesda, Maryland. In 2006, the groups reported that they had increased resolution by harnessing single-molecule photoactivatable fluorescent proteins and compiling images of thousands to millions of them" They called the approach photoactivated localization microscopy (PALM).

At Harvard University in Cambridge, Massachusetts, physicist Xiaowei Zhuang has developed three-dimensional (3D) stochastic optical reconstruction microscopy (STORM), which uses photoswitchable probes to temporally separate the overlapping images of individual molecules and so boost resolution to ten times better than the diffraction limit.

Yet another approach fluorescence PALM (FPALM) was developed in 2006 by Samuel Hess, a physicist at the University of Maine in Orono. His group's technique involves looking at thousands of fluorophores at once, and localizing on small numbers at a time. These methods have already begun to demostrate their utility. For example, in 2007, Hess's group showed that FPALM could be used to detect proteins clustering in lipid rafts in living cells ${ }^{12}$. In 2008, Lippincott-Schwartz's group combined PALM with singleparticle tracking to detect the movement of membrane proteins in live cells ${ }^{13}$. And Zhuang's group used 3D STORM to image microtubules and other molecular structures within monkey kidney cells $^{14}$, later extending the method to multicolour 3D imaging of whole cells ${ }^{15}$. Hell's group, early in 2008 , used the STED method to show the movement of synaptic vesicles inside living neurons at video rate $^{16}$.

But the field is just warming up. "To people like me who were trained in physics or optics in the 1990 s, it's just unbelievable that one can image below the resolution of light," says Bernardo Sabatini, a neurobiologist at Harvard Medical School in Boston, Massachusetts. "The major revolution for the next 5 or 10 years is getting these advances to answer biological questions." 